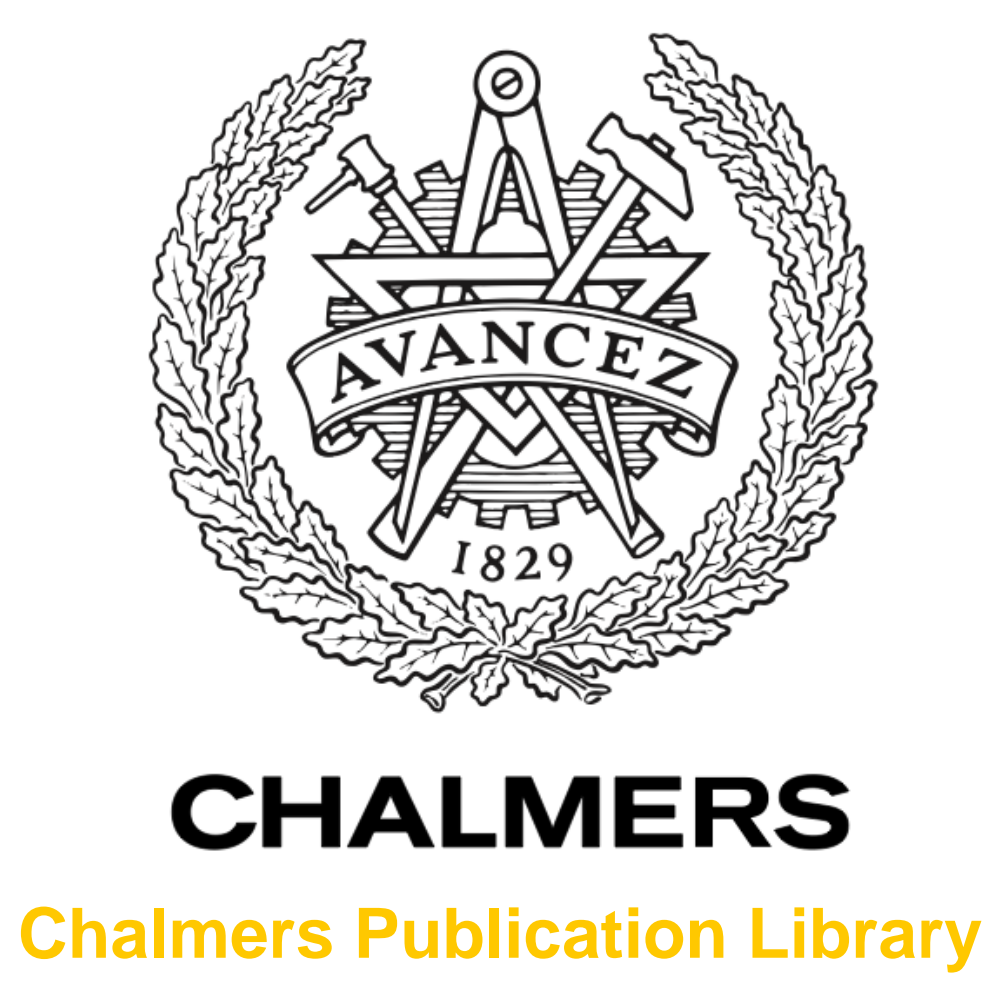

\title{
Performance Analysis of Centralized Relay Selection with Unreliable Control Information
}

This document has been downloaded from Chalmers Publication Library (CPL). It is the author's version of a work that was accepted for publication in:

IEEE Vehicular Technology Conference, VTC 2012-Fall, Quebec City, Canada, Sep. 2012

(ISSN: 1090-3038)

Citation for the published paper:

Papadogiannis, A. ; Svensson, T. (2012) "Performance Analysis of Centralized Relay Selection with Unreliable Control Information". IEEE Vehicular Technology Conference, VTC 2012-Fall, Quebec City, Canada, Sep. 2012

http://dx.doi.org/10.1109/VTCFall.2012.6399278

Downloaded from: http://publications.lib.chalmers.se/publication/156861

Notice: Changes introduced as a result of publishing processes such as copy-editing and formatting may not be reflected in this document. For a definitive version of this work, please refer to the published source. Please note that access to the published version might require a subscription.

Chalmers Publication Library (CPL) offers the possibility of retrieving research publications produced at Chalmers University of Technology. It covers all types of publications: articles, dissertations, licentiate theses, masters theses, conference papers, reports etc. Since 2006 it is the official tool for Chalmers official publication statistics. To ensure that Chalmers research results are disseminated as widely as possible, an Open Access Policy has been adopted.

The CPL service is administrated and maintained by Chalmers Library. 


\title{
Performance Analysis of Centralized Relay Selection with Unreliable Control Information
}

\author{
Agisilaos Papadogiannis and Tommy Svensson \\ Department of Signals and Systems, Chalmers University of Technology, Gothenburg, Sweden \\ Email: \{agisilaos.papadogiannis, tommy.svensson\}@ chalmers.se
}

\begin{abstract}
The potential of relay selection (RS) has been well appreciated. However if the control channels, conveying information on the selected relay node ( $R N)$, are unreliable, the gains of RS diminish. This is because the transmitting RN might not be the one maximizing the employed utility function. Furthermore, more than one of the RNs might end up transmitting, an event that could be performance degrading. It is also likely that no RN transmits, which is very undesirable. In this paper we assume decode-and-forward and present closed form expressions for the outage probability (OP) under Rayleigh fading when two potential RNs are available and the control information related to $\mathrm{RS}$ is corrupted by errors. We show that when control channels are unreliable, the OP performance degrades significantly and reaches a floor as the signal-to-noise ratio of the RN-to-destination data channel grows. Furthermore when control channels are very unreliable, suboptimal schemes not requiring control information, like repetition coding, outperform RS.
\end{abstract}

Index Terms-Relay selection, control channels, decode-andforward (DF), performance analysis.

\section{INTRODUCTION}

The use of relay nodes (RNs) is shown to greatly benefit wireless systems and their importance for future networks has been well recognized [1], [2]. RNs can bring significant diversity gains as well as other benefits to wireless networks without requiring costly multi-antenna nodes [3]. A spectrally efficient way to utilize RNs is through single relay selection (RS), where the RN optimizing a given metric is chosen to transmit amongst the available RNs [4]-[7].

Centralized RS requires that information on the chosen RN is reliably distributed to all $\mathrm{RNs}$ in order for each $\mathrm{RN}$ to know whether it should transmit or remain silent. Therefore the effectiveness of RS heavily relies on the reliability of the control channels between the entity performing RS and the RNs. As shown in [8], [9], control channels introduce errors which can greatly degrade network performance. [4] [7] are dedicated to analyzing and evaluating performance of RS. However to the best of the authors' knowledge the RS performance has not been studied as a function of the reliability of the control information.

In this paper we consider a source node that communicates with a destination node with the aid of two RNs employing decode-and-forward (DF). A central network entity $(\mathrm{CNE})^{1}$ collects estimates of the channels between the destination

\footnotetext{
${ }^{1}$ The CNE is a logical entity that can be implemented for instance at the destination or the source node.
}

and the RNs and selects the RN providing maximum signalto-noise ratio (SNR). The index of the selected $\mathrm{RN}$ is fed back by the CNE to the RNs through control channels that introduce errors. In this paper we consider two potential RNs and we model control channels as a two-input four-output binary asymmetric channel (BAC). We derive closed-form expressions for the outage probability (OP) under Rayleigh fading as a function of the error probability of the control information. We show that unreliable control information results in a degraded OP performance; OP reaches a floor as the SNR of the relay-to-destination data channel grows. It is also shown that when control channels are very unreliable, it is preferable to switch-off RS and employ suboptimal schemes like repetition coding that are nonetheless robust as they do not rely on control information.

\section{SYSTEM MODEL}

We consider a system comprising a source node $S$, a destination node $D$ and two half-duplex RNs, $R_{k}, k=1,2$. It is assumed that there is no direct $S \rightarrow D$ connection, i.e., $S$ communicates with $D$ only via the RNs. $S$ transmits a symbol $s$ of energy $E_{s}$ and the RNs employ the DF protocol assuming they are always able to decode $s$. The channel coefficients of the $R_{k} \rightarrow D$ data links $h_{k}, k=1,2$ are modeled as independent and non-identically distributed (INID) zero mean complex Gaussian random variables (RVs), i.e., $h_{k} \sim \mathcal{C N}\left(0, \bar{\gamma}_{k} / 2\right)$, where $\bar{\gamma}_{k}=\mathbb{E}\left[\left|h_{k}\right|^{2}\right] \frac{E_{s}}{N_{0}}$ is the average SNR of the link. $N_{0}$ denotes the one side power spectral density of the zero-mean circularly symmetric additive white Gaussian noise and $\mathbb{E}[$. denotes expectation. The envelope $\left|h_{k}\right|$ of these RVs follows the Rayleigh distribution with probability distribution function (PDF) $f_{\left|h_{k}\right|}(x)=\frac{2 x}{\bar{\gamma}_{k}} e^{-x^{2} / \bar{\gamma}_{k}}$. The instantaneous SNRs of the $R_{k} \rightarrow D$ links, $\gamma_{k}=\left|h_{k}\right|^{2} \frac{E_{s}}{N_{0}}$, are exponentially distributed RVs whose cumulative distribution function (CDF) is

$$
F_{\gamma_{k}}(x)=1-e^{-\lambda_{k} x}, \text { where } \lambda_{\mathrm{k}}=\frac{1}{\bar{\gamma}_{\mathrm{k}}} .
$$

The probability density function (PDF) of these RVs is $f_{\gamma_{k}}(x) \triangleq d F_{\gamma_{k}}(x) / d x=\lambda_{k} e^{-\lambda_{k} x}$.

\section{Performance ANALYsis}

In this section we model the control channel for RS and analyze the OP performance as a function of the error probabilities of the control channel. To compare the achievable performance we employ repetition coding (REP) with selection combining 
(SC) or maximal ratio combining (MRC). This is a suboptimal scheme that requires no control information and acts as our baseline.

\section{A. Repetition Coding}

The simplest transmission scheme would be to allow the RNs to transmit the decoded source symbol to $D$ in a repetitive fashion, one after the other in three time slots [3]. In the first slot, $S$ transmits a symbol $s$ to RNs which decode it. In the second slot, $R_{1}$ forwards $s$ to $D$ while $R_{2}$ remains silent. In the third slot, $R_{2}$ forwards $s$ to $D$, while $R_{1}$ remains silent. $D$ performs diversity combining, e.g., MRC or the simpler SC.

1) Selection Combining: For the $\mathrm{SC}$ case, $D$ decodes the signal of the RN providing the highest SNR [3]

$$
\gamma_{\mathrm{SC}}=\gamma_{\max }=\max \left\{\gamma_{1}, \gamma_{2}\right\} .
$$

Using the mutual information definition, the OP for a source transmit rate of $\mathcal{R}$ bits $/ \mathrm{s} / \mathrm{Hz}$ is

$$
\begin{aligned}
P_{\text {out }}^{R E P-S C}(\mathcal{R}) & =\operatorname{Pr}\left[\mathcal{R}>\frac{1}{3} \log _{2}\left(1+\gamma_{\max }\right)\right] \\
& =\operatorname{Pr}\left[\gamma_{\max }<\gamma_{\mathrm{rep}}\right]=F_{\gamma_{\max }}\left(\gamma_{\mathrm{rep}}\right),
\end{aligned}
$$

where $\gamma_{\text {rep }}=2^{3 \mathcal{R}}-1$ and $F_{\gamma_{\max }}(x)$ is the CDF of the maximum of $\gamma_{1}$ and $\gamma_{2}$. The pre-log factor $\frac{1}{3}$ of the mutual information expression comes from the fact that repetitive transmission requires three time slots. The $\mathrm{CDF}$ of the maximum of a set of $K$ RVs $\gamma_{1}, \gamma_{2}, \ldots, \gamma_{K}$, with $\lambda_{k}=\frac{1}{\bar{\gamma}_{k}}$ for $k=1,2, \ldots, K$, equals the product of their individual $\mathrm{CDFs}$ [10], i.e., $F_{\gamma_{\max }}(x)=\prod_{k=1}^{K} F_{\gamma_{k}}(x)$. In the considered case of two exponentially distributed RVs, this results in

$$
F_{\gamma_{\mathrm{SC}}}(x)=\left(1-e^{-\lambda_{1} x}\right)\left(1-e^{-\lambda_{2} x}\right) .
$$

By plugging (4) into (3), the OP of REP-SC scheme is $P_{\text {out }}^{R E P-S C}\left(\gamma_{\text {rep }}\right)=\left(1-e^{-\lambda_{1} \gamma_{\text {rep }}}\right)\left(1-e^{-\lambda_{2} \gamma_{\text {rep }}}\right)$.

2) Maximal Ratio Combining: For the MRC case it is assumed that $D$ has perfect channel state information (CSI) of the $R_{k} \rightarrow D$ links, where $k=1,2$. The end SNR after the signal combining is [11]

$$
\gamma_{\mathrm{MRC}}=\gamma_{1}+\gamma_{2} .
$$

To obtain the CDF of $\gamma_{\mathrm{MRC}}$ we use the the moment-generating function (MGF) approach [10]. By definition, the MGF of $\gamma_{k}$ is

$$
\mathcal{M}_{\gamma_{k}}(s)=\int_{0}^{+\infty} e^{-s x} f_{\gamma_{k}}(x) d x=\frac{\lambda_{k}}{s+\lambda_{k}} .
$$

As $\gamma_{1}$ and $\gamma_{2}$ are independent, the MGF of $\gamma_{\mathrm{MRC}}$ equals $\mathcal{M}_{\gamma_{\mathrm{MRC}}}(s)=\mathcal{M}_{\gamma_{1}}(s) \mathcal{M}_{\gamma_{2}}(s)$. Therefore

$$
\mathcal{M}_{\gamma_{\mathrm{MRC}}}(s)=\frac{\lambda_{1} \lambda_{2}}{\left(\lambda_{1}+s\right)\left(\lambda_{2}+s\right)} .
$$

The CDF of $\gamma_{\mathrm{MRC}}$ can be obtained as follows

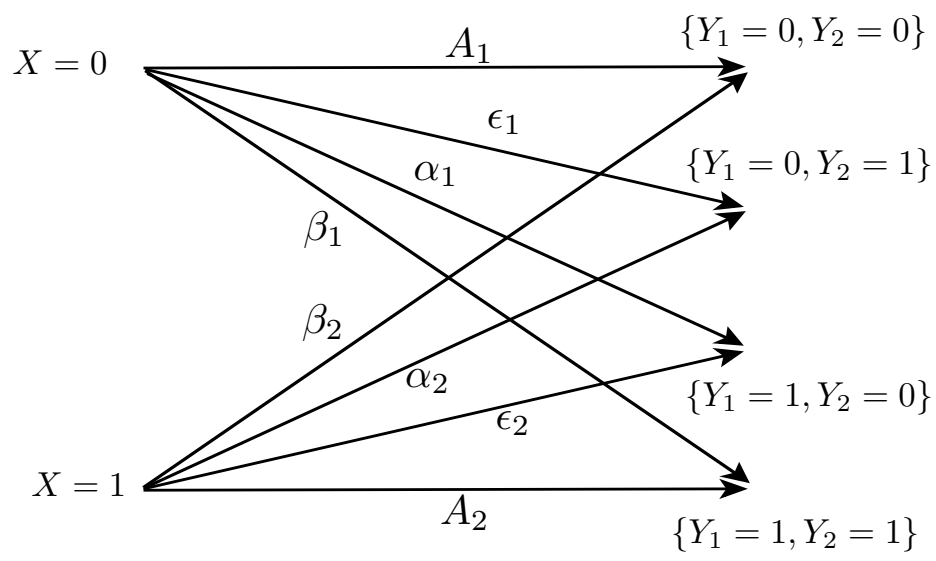

Fig. 1. An illustration of the control channels between CNE and $R_{1}, R_{2}$ which are modeled as a two-input four-output binary asymmetric channel (BAC).

$$
F_{\gamma_{\mathrm{MRC}}}(x)=\mathbb{L}^{-1}\left\{\frac{\mathcal{M}_{\gamma_{\mathrm{MRC}}}(s)}{s} ; x\right\},
$$

where $\mathbb{L}^{-1}\{.,$.$\} denotes the inverse Laplace transform. From$ (7) and (8) and after some manipulation, we obtain

$$
F_{\gamma_{\mathrm{MRC}}}(x)= \begin{cases}1+\frac{\lambda_{2} e^{-\lambda_{1} x}}{\lambda_{1}-\lambda_{2}}+\frac{\lambda_{1} e^{-\lambda_{2} x}}{\lambda_{2}-\lambda_{1}}, & \text { if } \lambda_{1} \neq \lambda_{2} \\ 1-\left(1+\lambda_{1} x\right) e^{-\lambda_{1} x}, & \text { if } \lambda_{1}=\lambda_{2}\end{cases}
$$

The resulting OP for this scheme can be obtained directly from the above CDF, i.e., $P_{\text {out }}^{R E P-S C}\left(\gamma_{\text {rep }}\right)=F_{\gamma_{\mathrm{MRC}}}\left(\gamma_{\mathrm{rep}}\right)$.

\section{B. Relay Selection with Unreliable Control Information}

As previously discussed, repetition coding with two RNs requires three time slots, a fact that limits performance. A more efficient scheme is to allow the CNE select a single RN for transmission, the one providing the maximum $R_{k} \rightarrow D$ SNR. This scheme requires two time slots for transmission, which provides a significant advantage compared to repetition coding as the capacity pre-log factor in this case is only $\frac{1}{2}$. Without loss of generality we assume that the CNE is implemented at $D$. Since two RNs are assumed, let $X \in\{0,1\}$ be a discrete $\mathrm{RV}$ representing the index of the selected $\mathrm{RN}$ which is fed back by the CNE to $R_{1}$ and $R_{2}$ through the control channels, where

$$
\begin{aligned}
& \operatorname{Pr}[X=0]=\mathbb{E}_{\gamma_{1}}\left\{\operatorname{Pr}\left[\gamma_{2}<\gamma_{1} \mid \gamma_{1}\right]\right\} \\
& \operatorname{Pr}[X=1]=1-\operatorname{Pr}[X=0],
\end{aligned}
$$

where $\mathbb{E}\{$.$\} denotes expectation and \operatorname{Pr}[$.$] denotes probability.$ After some manipulation, we obtain the expression of (11) for $\operatorname{Pr}[X=0]$.

Let $Y_{1}, Y_{2} \in\{0,1\}$ be the indices of the selected $\mathrm{RN}$ as received by $R_{1}$ and $R_{2}$ respectively. The control channels from CNE to $R_{1}$ and $R_{2}$ are assumed to be unreliable, i.e., $X$ might 


$$
\operatorname{Pr}[X=0]=\mathbb{E}_{\gamma_{1}}\left\{\operatorname{Pr}\left[\gamma_{2}<\gamma_{1} \mid \gamma_{1}\right]\right\}=\mathbb{E}_{\gamma_{1}}\left\{F_{\gamma_{2}}\left(\gamma_{1}\right)\right\}=\int_{0}^{+\infty}\left(1-e^{-\lambda_{2} \gamma_{1}}\right) \lambda_{1} e^{-\lambda_{1} \gamma_{1}} d \gamma_{1}=1-\frac{\lambda_{1}}{\lambda_{1}+\lambda_{2}}
$$

not be decoded correctly by $R_{1}$ and/or $R_{2}$ and this can prevent the RN with the best SNR to $D$ from transmitting. Regarding transmission, the following four events can occur:

1) Event $\mathcal{E}_{1}=\left\{\gamma_{\mathrm{RS}}=\gamma_{\max }=\max \left\{\gamma_{1}, \gamma_{2}\right\}\right\}$ : the $\mathrm{RN}$ providing the maximum SNR transmits (desirable event).

2) Event $\mathcal{E}_{2}=\left\{\gamma_{\mathrm{RS}}=\gamma_{\min }=\min \left\{\gamma_{1}, \gamma_{2}\right\}\right\}$ : the $\mathrm{RN}$ providing the minimum SNR transmits.

3) Event $\mathcal{E}_{3}=\left\{\gamma_{\mathrm{RS}}=\gamma_{\text {sum }}=\left|h_{1}+h_{2}\right|^{2}\right\}$ : both RNs transmit simultaneously and independently.

4) Event $\mathcal{E}_{4}=\left\{\gamma_{\mathrm{RS}}=0\right\}$ : no RN transmits.

Therefore, the CDF of RS with unreliable control information can be expressed as

$$
\begin{aligned}
F_{\gamma_{\mathrm{RS}}}(x)=\operatorname{Pr}\left[\mathcal{E}_{1}\right] & F_{\gamma_{\text {max }}}(x)+\operatorname{Pr}\left[\mathcal{E}_{2}\right] F_{\gamma_{\min }}(x) \\
& +\operatorname{Pr}\left[\mathcal{E}_{3}\right] F_{\gamma_{\text {sum }}}(x)+\operatorname{Pr}\left[\mathcal{E}_{4}\right] .
\end{aligned}
$$

For a source transmit rate of $\mathcal{R}$ bits $/ \mathrm{s} / \mathrm{Hz}$ the OP when feedback is unreliable is $P_{\text {out }}^{R S}\left(\gamma_{\mathrm{rs}}\right)=F_{\gamma_{\mathrm{RS}}}\left(\gamma_{\mathrm{rs}}\right)$, where $\gamma_{r s}=2^{2 \mathcal{R}}-1$.Therefore in order to express the above CDF, and thus the related OP, in closed form it is necessary to evaluate the probabilities of occurrence of the above events and to express the related CDFs $F_{\gamma_{\min }}(x)$ and $F_{\gamma_{\text {sum }}}(x)$, as $F_{\gamma_{\max }}(x)$ is given by (4). The minimum of a set of $K$ exponentially distributed RVs $\gamma_{2}, \gamma_{2}, \ldots, \gamma_{K}$, with $\lambda_{k}=\frac{1}{\bar{\gamma}_{k}}$ for $k=1,2, \ldots, K$, is also exponentially distributed with the following CDF [12, ch. (4.6)]

$$
F_{\gamma_{\min }}(x)=1-e^{-\left(\sum_{k=1}^{K} \lambda_{k}\right) x}
$$

The $\mathrm{RV} \gamma_{\text {sum }}=\left|\sum_{k=1}^{K} h_{k}\right|^{2}$ where $h_{k} \sim \mathcal{C N}\left(0, \bar{\gamma}_{k} / 2\right)$, is exponentially distributed with the following $\mathrm{CDF}$

$$
F_{\gamma_{\text {sum }}}(x)=1-e^{-\lambda_{\text {sum }} x}
$$

where $\lambda_{\text {sum }}=\frac{\sum_{k=1}^{K} \lambda_{k}}{\prod_{k=1}^{K} \lambda_{k}}[12$, ch. (4.6)]. For simplifying the notation, let $C_{1} \stackrel{=}{=}\left(1-e^{-\lambda_{1} \gamma_{\mathrm{rs}}}\right)\left(1-e^{-\lambda_{2} \gamma_{\mathrm{rs}}}\right), C_{2}=1-$ $e^{-\left(\lambda_{1}+\lambda_{2}\right) \gamma_{\mathrm{rs}}}$, and $C_{3}=1-e^{-\left(\frac{\lambda_{1}+\lambda_{2}}{\lambda_{1} \lambda_{2}}\right) \gamma_{\mathrm{rs}}}$.

Fig. 1 illustrates the control channel between CNE and $R_{1}, R_{2}$ which is modeled as a two-input four-output BAC. The conditional probabilities of the eight different states of the system are as follows

$$
\begin{aligned}
& \operatorname{Pr}\left[Y_{1}=0, Y_{2}=0 \mid X=0\right]=\operatorname{Pr}\left[\mathcal{E}_{1} \mid X=0\right]=A_{1} \\
& \operatorname{Pr}\left[Y_{1}=1, Y_{2}=1 \mid X=1\right]=\operatorname{Pr}\left[\mathcal{E}_{1} \mid X=1\right]=A_{2} \\
& \operatorname{Pr}\left[Y_{1}=1, Y_{2}=1 \mid X=0\right]=\operatorname{Pr}\left[\mathcal{E}_{2} \mid X=0\right]=\beta_{1} \\
& \operatorname{Pr}\left[Y_{1}=0, Y_{2}=0 \mid X=1\right]=\operatorname{Pr}\left[\mathcal{E}_{2} \mid X=1\right]=\beta_{2} \\
& \operatorname{Pr}\left[Y_{1}=0, Y_{2}=1 \mid X=0\right]=\operatorname{Pr}\left[\mathcal{E}_{3} \mid X=0\right]=\epsilon_{1} \\
& \operatorname{Pr}\left[Y_{1}=0, Y_{2}=1 \mid X=1\right]=\operatorname{Pr}\left[\mathcal{E}_{3} \mid X=1\right]=\alpha_{2} \\
& \operatorname{Pr}\left[Y_{1}=1, Y_{2}=0 \mid X=0\right]=\operatorname{Pr}\left[\mathcal{E}_{4} \mid X=0\right]=\alpha_{1} \\
& \operatorname{Pr}\left[Y_{1}=1, Y_{2}=0 \mid X=1\right]=\operatorname{Pr}\left[\mathcal{E}_{4} \mid X=1\right]=\epsilon_{2},
\end{aligned}
$$

where $A_{1}=1-\epsilon_{1}-\alpha_{1}-\beta_{1}$ and $A_{2}=1-\epsilon_{2}-\alpha_{2}-\beta_{2}$. Regarding the probabilities of transmission events, from the law of total probability it follows that for $k=1,2,3,4$

$$
\operatorname{Pr}\left[\mathcal{E}_{k}\right]=\sum_{x \in\{0,1\}} \operatorname{Pr}\left[\mathcal{E}_{k} \mid X=x\right] \operatorname{Pr}[X=x] .
$$

Therefore by plugging (15) and (10) into (16) we obtain the following expressions for the transmission event probabilities

$$
\begin{aligned}
& \operatorname{Pr}\left[\mathcal{E}_{1}\right]=A_{1}\left(1-\frac{\lambda_{1}}{\lambda_{1}+\lambda_{2}}\right)+A_{2} \frac{\lambda_{1}}{\lambda_{1}+\lambda_{2}} \\
& \operatorname{Pr}\left[\mathcal{E}_{2}\right]=\beta_{1}\left(1-\frac{\lambda_{1}}{\lambda_{1}+\lambda_{2}}\right)+\beta_{2} \frac{\lambda_{1}}{\lambda_{1}+\lambda_{2}} \\
& \operatorname{Pr}\left[\mathcal{E}_{3}\right]=\epsilon_{1}\left(1-\frac{\lambda_{1}}{\lambda_{1}+\lambda_{2}}\right)+\alpha_{2} \frac{\lambda_{1}}{\lambda_{1}+\lambda_{2}} \\
& \operatorname{Pr}\left[\mathcal{E}_{4}\right]=\alpha_{1}\left(1-\frac{\lambda_{1}}{\lambda_{1}+\lambda_{2}}\right)+\epsilon_{2} \frac{\lambda_{1}}{\lambda_{1}+\lambda_{2}} .
\end{aligned}
$$

To express the CDF of RS as a function of the error probabilities, we plug the values for the transmission event probabilities of (17) and the CDFs given by (4), (13) and (14) into (12) yielding the OP expression of (18). After some algebraic manipulation we obtain the final expression (19) for $P_{\text {out }}^{R S}\left(\gamma_{\mathrm{rs}}\right)$, where

$$
\begin{aligned}
K_{1} & =A_{1} C_{1}+\beta_{2} C_{2}+\epsilon_{1} C_{3}+\alpha_{1} \\
K_{2} & =C_{1}\left(A_{2}-A_{1}\right)+C_{2}\left(\beta_{2}-\beta_{1}\right) \\
& +C_{3}\left(\alpha_{2}-\epsilon_{1}\right)+\epsilon_{2}-\alpha_{1} .
\end{aligned}
$$

Outage Probability Lower Bound: For the ideal case where control information is always reliable, we obtain the following lower bound for the OP of RS

$$
P_{\text {out }}^{R S-L B}(\mathcal{R})=\lim _{\left\{A_{1}, A_{2}\right\} \rightarrow 1}\left[K_{1}+\frac{K_{2} \lambda_{1}}{\lambda_{1}+\lambda_{2}}\right]=C_{1} .
$$




$$
\begin{array}{r}
P_{\mathrm{out}}^{R S}\left(\gamma_{\mathrm{rs}}\right)=F_{\gamma_{\mathrm{RS}}}\left(\gamma_{\mathrm{rs}}\right)=A_{1}\left(1-\frac{\lambda_{1}}{\lambda_{1}+\lambda_{2}}\right) C_{1}+A_{2} \frac{\lambda_{1}}{\lambda_{1}+\lambda_{2}} C_{1}+\beta_{1}\left(1-\frac{\lambda_{1}}{\lambda_{1}+\lambda_{2}}\right) C_{2}+\beta_{2} \frac{\lambda_{1}}{\lambda_{1}+\lambda_{2}} C_{2}+ \\
\epsilon_{1}\left(1-\frac{\lambda_{1}}{\lambda_{1}+\lambda_{2}}\right) C_{3}+\alpha_{2} \frac{\lambda_{1}}{\lambda_{1}+\lambda_{2}} C_{3}+\alpha_{1}\left(1-\frac{\lambda_{1}}{\lambda_{1}+\lambda_{2}}\right)+\epsilon_{2} \frac{\lambda_{1}}{\lambda_{1}+\lambda_{2}} . \\
\begin{array}{r}
P_{\mathrm{out}}^{R S}\left(\gamma_{\mathrm{rs}}\right)=\left[A_{1} C_{1}+\beta_{1} C_{2}+\epsilon_{1} C_{3}+\alpha_{1}\right]+\frac{\lambda_{1}}{\lambda_{1}+\lambda_{2}}\left[C_{1}\left(A_{2}-A_{1}\right)+C_{2}\left(\beta_{2}-\beta_{1}\right)+C_{3}\left(\alpha_{2}-\epsilon_{1}\right)+\epsilon_{2}-\alpha_{1}\right] \\
=K_{1}+K_{2} \frac{\lambda_{1}}{\lambda_{1}+\lambda_{2}} .
\end{array}
\end{array}
$$

It can be observed that $P_{\text {out }}^{R S-L B}(\mathcal{R})<P_{\text {out }}^{R E P-M R C}(\mathcal{R})<$ $P_{\text {out }}^{R E P-S C}(\mathcal{R})$, showing that RS with reliable control information is always more efficient than REP in terms of OP.

\section{Performance eVAluation}

To evaluate the OP performance of RS under unreliable control information we need to express the transition probabilities of (15). Let $P_{e, 1}=\operatorname{Pr}\left[Y_{1} \neq X\right]$ and $P_{e, 2}=\operatorname{Pr}\left[Y_{2} \neq X\right]$ be the bit error probabilities on the control links $\mathrm{CNE} \rightarrow \mathrm{R}_{1}$ and $\mathrm{CNE} \rightarrow \mathrm{R}_{2}$ respectively, which are assumed to be independent, i.e., $\operatorname{Pr}\left[Y_{1} \neq X, Y_{2} \neq X\right]=P_{e, 1} P_{e, 2}$. In this case the values of (15) can be expressed in terms of $P_{e, 1}$ and $P_{e, 2}$ as follows

$$
\begin{gathered}
\epsilon_{1}=\epsilon_{2}=\left(1-P_{e, 1}\right) P_{e, 2} \\
\alpha_{1}=\alpha_{2}=P_{e, 1}\left(1-P_{e, 2}\right) \\
\beta_{1}=\beta_{2}=P_{e, 1} P_{e, 2} .
\end{gathered}
$$

$\epsilon_{1}$ and $\epsilon_{2}$ take the value of (22a) as they represent the event when the control bit $X$ is decoded correctly by $R_{1}$ and incorrectly by $R_{2}, \alpha_{1}$ and $\alpha_{2}$ take the value of (22b) as the bit is decoded incorrectly by $R_{1}$ and correctly by $R_{2}$, and $\beta_{1}$ and $\beta_{2}$ take the value of $(22 \mathrm{c})$ as the bit is decoded incorrectly by both $R_{1}$ and $R_{2}$.

For the present evaluation we consider that the $R_{1} \rightarrow D$ and $R_{2} \rightarrow D$ data channels are independent and identically distributed. The analytical results are validated by Monte Carlo simulations and it can be seen that there is perfect match. Fig. 2 plots the achieved OP versus the average SNR $\bar{\gamma}_{k}=E_{s} / N_{0}$ of the $R_{k} \rightarrow D$ data channels, where $k=1,2$, for the baseline repetition coding scheme with SC (REP-SC) and MRC (REP-MRC), the lower bound of RS (RS-LB) and the RS with unreliable control information. For the latter case we plot the OP for $P_{e}=P_{e, 1}=P_{e, 2}=10^{-1}, P_{e}=10^{-2}$ and $P_{e}=10^{-3}$. It is clear that as the data channel average SNR $\bar{\gamma}_{k}$ grows, the errors introduced by the unreliable control channel degrade the OP performance significantly and result in a floor for OP. It can be seen that when SNR exceeds $18 \mathrm{~dB}$, even the simple REP-SC achieves lower OP than RS when $P_{e}$ is higher than $10^{-2}$. Fig. 3 plots the OP versus $P_{e}$ for all considered schemes when $\bar{\gamma}_{1}=\bar{\gamma}_{2}=10 \mathrm{~dB}$. For this SNR, although the OP of RS increases as $P_{e}$ grows, RS achieves better performance than both REP schemes. Fig. 4 plots OP versus $P_{e}$ for $\bar{\gamma}_{1}=\bar{\gamma}_{2}=20 \mathrm{~dB}$ showing that in

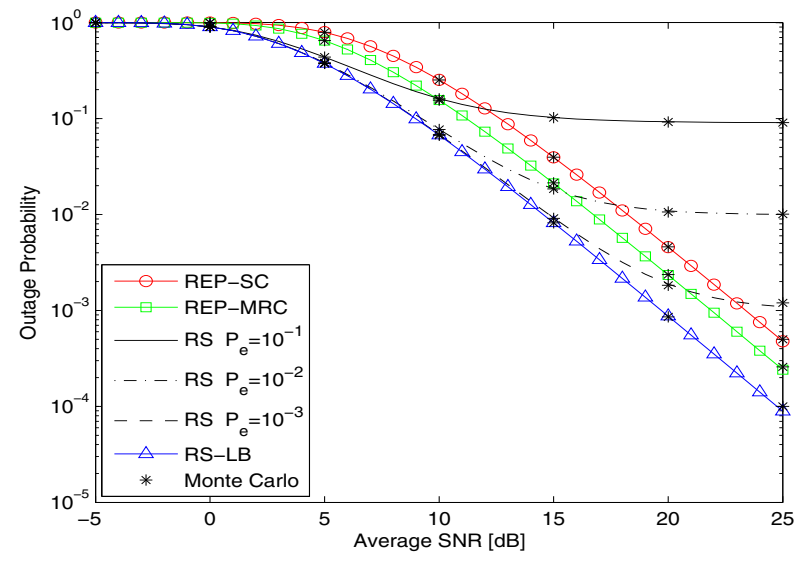

Fig. 2. Outage probability versus the average data channel SNR $\bar{\gamma}_{k}$ when $\mathcal{R}=1 \mathrm{bit} / \mathrm{s} / \mathrm{Hz}$ and $P_{e}=P_{e, 1}=P_{e, 2}$.

this high SNR regime REP-SC outperforms RS when $P_{e}$ is greater than $10^{-2.6}$, while REP-MRC outperforms RS when $P_{e}$ is greater than $10^{-2.9}$. It should be noted that although the schemes that do not rely on control information, i.e., REPSC and REP-MRC are suboptimal, they outperform RS when control channels are unreliable. This shows the importance of the quality of control information.

Figs. 5 and 6 plot the OP of the RS scheme as a function of the error probabilities of the control channels $P_{e, 1}$ and $P_{e, 2}$ when the average SNR of the data channels $\bar{\gamma}_{k}$ is 10 and $20 \mathrm{~dB}$ respectively and $\mathcal{R}=1 \mathrm{bit} / \mathrm{s} / \mathrm{Hz}$. These plots show how the difference between $P_{e, 1}$ and $P_{e, 2}$ affects performance. More specifically, for $\bar{\gamma}_{1}=\bar{\gamma}_{2}=10 \mathrm{~dB}$ (medium SNR case), the difference between $P_{e, 1}$ and $P_{e, 2}$ does not have very high impact on OP. However, in the high SNR of 20 $\mathrm{dB}$ and when $P_{e, 1}$ and $P_{e, 2}$ take high values (above 0.4), the more similar the values of $P_{e, 1}$ and $P_{e, 2}$ are, the lower the achieved OP. This trend shows that when control channels are highly unreliable, performance is better when the degree of unreliability is similar across control channels.

\section{CONClusion}

In this paper we investigated the impact of the reliability of control information on the performance of relay selection. To this end we derived closed-form expressions for the outage probability of single relay selection when the control channels 


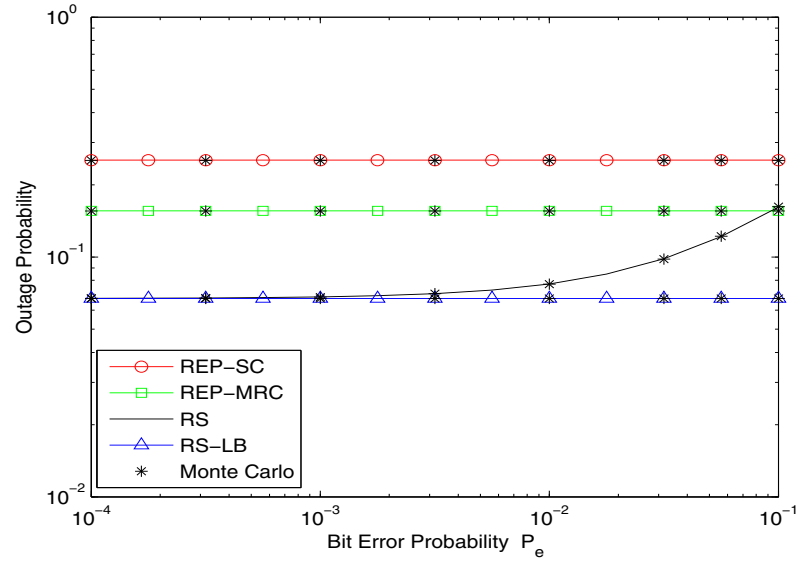

Fig. 3. Outage probability versus control channel bit error probability $P_{e}=$ $P_{e, 1}=P_{e, 2}$ when $\mathcal{R}=1 \mathrm{bit} / \mathrm{s} / \mathrm{Hz}$ and $\bar{\gamma}_{1}=\bar{\gamma}_{2}=10 \mathrm{~dB}$.

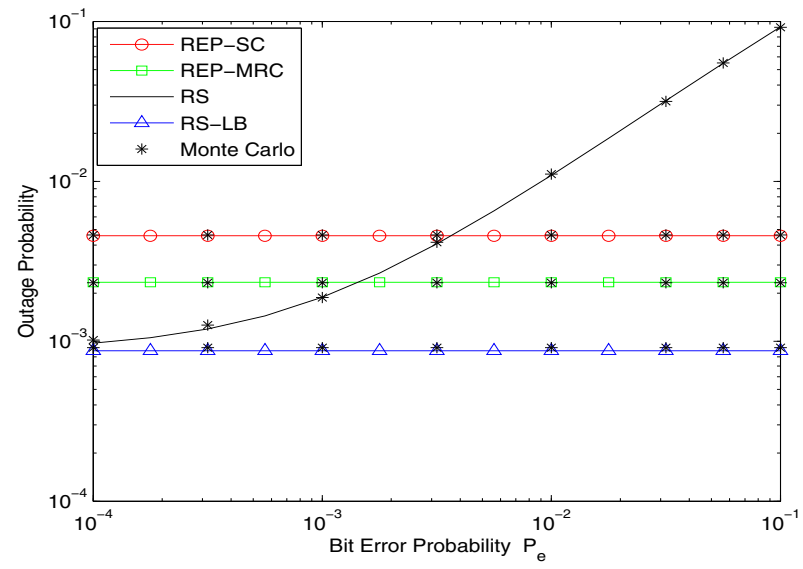

Fig. 4. Outage probability versus control channel bit error probability $P_{e}=$ $P_{e, 1}=P_{e, 2}$ when $\mathcal{R}=1 \mathrm{bit} / \mathrm{s} / \mathrm{Hz}$ and $\bar{\gamma}_{1}=\bar{\gamma}_{2}=20 \mathrm{~dB}$.

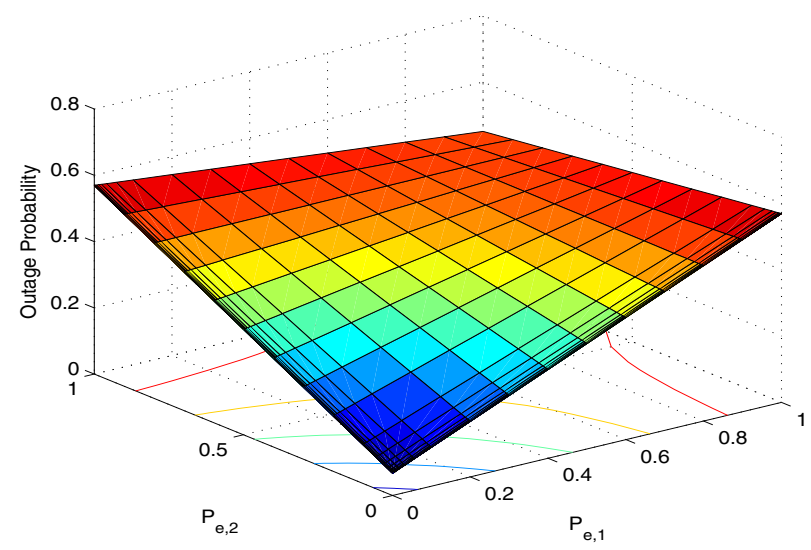

Fig. 5. Outage probability of RS versus $P_{e, 1}$ and $P_{e, 2}$ when $\mathcal{R}=1 \mathrm{bit} / \mathrm{s} / \mathrm{Hz}$ and $\bar{\gamma}_{1}=\bar{\gamma}_{2}=10 \mathrm{~dB}$.

introduce errors. We can conclude that relay selection is beneficial when the control channels are of high reliability

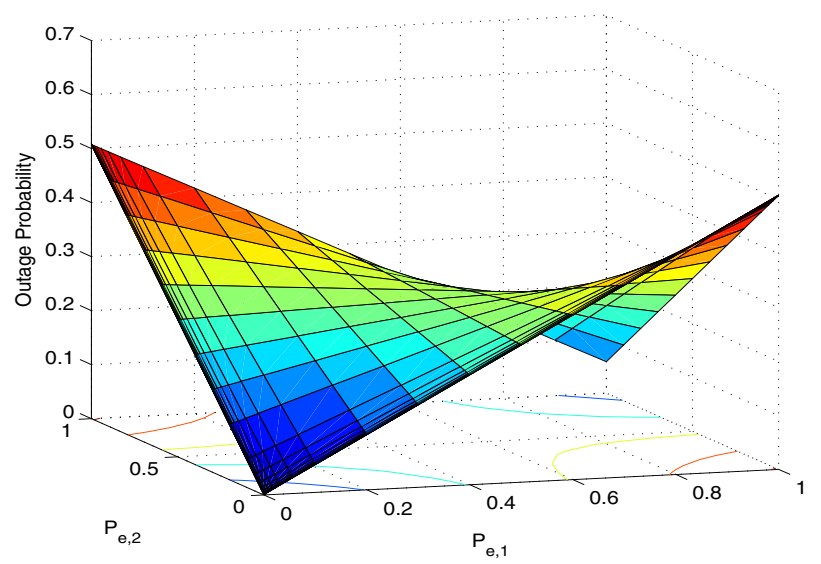

Fig. 6. Outage probability of RS versus $P_{e, 1}$ and $P_{e, 2}$ when $\mathcal{R}=1 \mathrm{bit} / \mathrm{s} / \mathrm{Hz}$ and $\bar{\gamma}_{1}=\bar{\gamma}_{2}=20 \mathrm{~dB}$.

or when the average data channel SNR is low. In high SNR and/or when the error probability in the control channels is high, suboptimal schemes like repetition coding, requiring no control information, are more robust.

\section{ACKNOWLEDGMENTS}

This work has been supported by the Swedish Research Council (VR), within the project 621-2009-4555 Dynamic Multipoint Wireless Transmission and the Swedish Agency for Innovation Systems (VINNOVA), within the project P36604-1 MAGIC

\section{REFERENCES}

[1] J. N. Laneman, D. N. C. Tse, and G. W. Wornell, "Cooperative diversity in wireless networks: efficient protocols and outage behavior," IEEE Trans. Inf. Theory, vol. 50, no. 12, pp. 3062-3080, Dec. 2004.

[2] S. Parkvall et al., "LTE-Advanced - Evolving LTE towards IMTAdvanced," in IEEE VTC 2008-Fall, Calgary, Canada, Sept. 2008.

[3] J. Hu and N. C. Beaulieu, "Performance analysis of decode-and-forward relaying with selection combining," IEEE Commun. Lett., vol. 11, no. 6 , pp. 489-491, June 2007.

[4] E. Beres and R. Adve, "Selection cooperation in multi-source cooperative networks," IEEE Trans. Wireless Commun., vol. 7, no. 1, pp. 118 -127 , jan. 2008.

[5] S. S. Ikki and M. H. Ahmed, "Performance of multiple-relay cooperative diversity systems with best relay selection over rayleigh fading channels," EURASIP J. Wireless Commun. Netw., vol. 2008, p. 7, 2008.

[6] A. Papadogiannis, A. Saadani, and E. Hardouin, "Exploiting dynamic relays with limited overhead in cellular systems," in IEEE GLOBECOM 2009, Hawaii, USA, 30 Nov.-4 Dec. 2009.

[7] D. Michalopoulos and G. K. Karagiannidis, "Performance analysis of single relay selection in Rayleigh fading," IEEE Trans. Wireless Commun., vol. 7, no. 10, pp. 3718-3724, Oct. 2009.

[8] A. Papadogiannis, E. Hardouin, and D. Gesbert, "A framework for decentralising multi-cell cooperative processing on the downlink," in IEEE GLOBECOM 2008, New Orleans, USA, 30 Nov.-4 Dec. 2008.

[9] — - "Decentralising multicell cooperative processing: A novel robust framework," EURASIP J. Wireless Commun. Netw., vol. 2009, p. 10 , Aug. 2009.

[10] M. K. Simon and M. S. Alouini, Digital Communication over Fading Channels, 2nd ed. New York: Wiley, 2005.

[11] N. C. Beaulieu and J. Hu, "A closed-form expression for the outage probability of decode-and-forward relaying in dissimilar rayleigh fading channels," IEEE Commun. Lett., vol. 10, no. 12, pp. 813 -815, dec 2006.

[12] B. C. Arnold, N. Balakrishnan, and H. N. Nagaraja, A First Course in Order Statistics, 1st ed. SIAM, 2008. 